\title{
Renal complications in children with malignancies: single-centre experience
}

\author{
Ashraf Bakr'1, Youssef Al-Tonbary², Mohamed Sarhan², Atef El-Mougy' ${ }^{2}$, Ashraf Fouda² \\ 1. Department of pediatric nephrology, Mansoura University Children's Hospital, Mansoura Faculty of Medicine, Mansoura, \\ Egypt. 2. Mansoura University Children's Hospital, Mansoura, Egypt
}

Correspondence: Youssef Al-Tonbary. Address: Mansoura University Children's Hospital, Mansoura, Egypt. Telephone: 20-123-165-302. Fax: 20-502-262-307. E-mail: ytonbary@gmail.com

Received: May 6, 2012

DOI : $10.5430 /$ jhm.v2n3p1
Accepted: June 24, $2012 \quad$ Published: September 1, 2012

URL: http://dx.doi.org/10.5430/jhm.v2n3p1

\section{Abstract}

Background and aim of the work: Renal complications in cancer patients are serious and may cause more morbidity and mortality. Acute renal dysfunction may affect optimal cancer treatment by requiring a decrease in chemotherapy dosage or by contraindicating potentially curative treatment. Our study aimed to evaluate different types of renal involvement before and after treatment of childhood malignancies.

Patients and Methods: All oncology patients at Mansoura University Children's Hospital diagnosed with different types of malignancies in seven years period (January 2003 to December 2009) were retrospectively reviewed for renal involvement before or after treatment.

Results: We evaluated 954 pediatric patients with different types of malignancies. Patients encountered renal involvement were 152 (15.9\%). A total of 105 patients diagnosed with acute renal failure (ARF), 18 had renal masses, 3 had nephrotic syndrome and 26 had hemorrhagic cystitis.

As regard outcome of acute renal failure, 70 patients (66.7\%) showed complete recovery of renal function, while 27 patients died (25.7\%). The remaining 8 patients (7.6\%) were maintained on chronic replacement therapy.

Conclusions: Renal involvement in children with cancer is a common problem. ARF is the commonest renal complication and most severe. It results from various causes. Early prediction and anticipation of renal insult and prompt management may reduce the risk of renal injury. All oncologists should keep in mind that they are not treating cancer only, but they are treating patient with cancer and should save the kidney as much as possible.

\section{Key words}

Acute renal failure, Tumor lysis syndrome, Cancer, Children

\section{Introduction}

Advances in pediatric oncology and improved methods of treatment have resulted in significant reduction in mortality, leading to the increasing number of long-term survivors. Intensive chemotherapy may affect endocrine, cardiac, respiratory, nervous, and renal systems ${ }^{[1,2]}$. Acute renal failure (ARF) is a frequent complication of cancer and its treatments. ARF constitutes a major source of morbidity and mortality and can also result in patients with cancer being 
unable to receive optimal therapeutic management and supportive care. ARF is a serious complication in cancer patients that causes additional morbidity and mortality. Optimal doses of chemotherapy and supportive drugs are often restricted in these patients, which jeopardizes patient outcomes ${ }^{[3]}$.

\section{Aim of the study}

Our study aimed to evaluate different types of renal involvement before and after treatment of childhood malignancies, prognostic impact and the outcome of these complications on the long term outcome of treatment and survival.

\section{Patients and Methods}

This study was a retrospective evaluation of children with malignancies as regard different renal complications before or after treatment.

\subsection{Patients}

All oncology patients at Mansoura University Children's Hospital diagnosed with different types of malignancies in seven years period (January 2003 to the end of December 2009) were retrospectively reviewed for renal involvement before or after treatment. Our patients were treated with either chemotherapy or chemotherapy and subsequent bone marrow transplantation which were provided for 18 patients (autologous or allogeneic).

The diagnosis of malignancies was made according to standard pathological criteria (bone marrow aspiration or tissue biopsy). All age categories were included (range 1 month to 18 year); 590 patients were males and 364 were females (male: female ratio 1.6:1). No patient was in chronic renal failure or on hemodialysis, before malignancy was diagnosed.

\subsection{Renal complications}

Renal complications were diagnosed according to clinical and laboratory and pathological criteria. For patients without preexisting renal disease, normal values were $0.5-1.1 \mathrm{mg} / \mathrm{dL}$ for serum creatinine, and 10-50 mg/dL for serum urea. For the diagnosis of a renal complication, the laboratory values had to be abnormal for on at least 2 separate days. The onset of renal complications was also determined: at diagnosis before starting chemotherapy or after starting chemotherapy. A different time frame was used for patients who underwent bone marrow transplantation: before or during conditioning, during the first 100 days after transplantation, or later.

\section{Results}

In this study, we evaluated 954 pediatric patients with different types of malignancies. They were as follows: 317 patients had acute lymphoblastic leukemia (ALL) (33.2\%), 142 patients with non-Hodgkin lymphoma (NHL) (14.9\%), 114 with neuroblastoma (11.9\%), 86 with acute myeloid leukemia (AML) (9.9\%), 96 with Hodgkin's disease (HD) (10.0\%), while other types of malignancies represent the remaining $20 \%$ of the total patients (Table 1 ).

Out of the reviewed children with malignancies, 152 had renal involvement (15.9\%). Of them 105 patients had ARF (69.1\%), 18 patients had renal masses (11.8\%), 3 patients had nephrotic syndrome (2\%) and 26 patients were complicated with hemorrhagic cystitis (17.1\%) as shown in Table 2.

Thirty four patients (32.4\%) had ARF before chemotherapy was started and 71 patients (67.6\%) after chemotherapy. ARF due to pretreatment tumor lysis syndrome (TLS) occurred in 21 patients out of 33 patients with TLS. Majority of patients were of acute leukemia (14 ALL and 1 AML) and 6 patients had bulky abdominal NHL. Serum uric acid at diagnosis 
ranged from 11-55 $\mathrm{mg} / \mathrm{dL}$. Seven patients had post-renal failure (obstructive uropathy) due to tumor compression by retroperitoneal rhabdomyosarcoma ( 2 patients), germ cell tumor (1 patient), paraspinal neuroblastoma ( 2 patients), and Burkitt's lymphoma ( 2 patients). The other 6 patients with ARF were related to renal infiltration by leukemic blasts (2 patients), NHL (2 patients), and neuroblastoma (2 patients).

Table 1. Clinical data of the studied patients

\begin{tabular}{lll}
\hline & & Number (\%) \\
\hline Age (range) & 1month to 18 year & \\
Male/female & $590 / 364(1.6 / 1)$ & \\
Type of malignancy & Acute lymphoblastic leukemia (ALL) & $317(33.2 \%)$ \\
& Non Hodgkin's lymphoma (NHL) & $142(14.9 \%)$ \\
& Neuroblastoma & $114(11.9 \%)$ \\
& Hodgkin disease (HD) & $96(10.0 \%)$ \\
& Acute myeloid leukemia (AML) & $86(9.9 \%)$ \\
& Rhabdomyosarcoma & $35(3.6 \%)$ \\
& Wilm's tumor & $33(3.5 \%)$ \\
& Brain tumor & $25(2.6 \%)$ \\
& Osteosarcoma & $25(2.6 \%)$ \\
& Ewing sarcoma & $20(2.0 \%)$ \\
Chronic myeloid leukemia & $20(2.0 \%)$ \\
Langerhans cell histiocytosis & $19(1.9 \%)$ \\
Hepatoblastoma & $10(1 \%)$ \\
Germ cell tumor & $10(1 \%)$ \\
Retinoblastoma & $2(<1 \%)$ \\
Total number & $954(100 \%)$ \\
\hline
\end{tabular}

ARF occurring after starting chemotherapy occurred in 71 patients $(67.6 \%)$ was due to: TLS (12 patients), drug related (15 patients) and pre-renal failure due to severe volume depletion (29 patients) and sepsis or septic shock (15 patients).

Table 2. Forms of renal affection in different types of childhood malignancies

\begin{tabular}{|c|c|c|c|c|}
\hline $\begin{array}{l}\text { Patients with renal } \\
\text { involvement }\end{array}$ & \multicolumn{2}{|c|}{ Types of renal involvement } & No (\%) & \\
\hline \multirow{9}{*}{$152 / 954(15.9 \%)$} & \multirow{6}{*}{\multicolumn{2}{|c|}{$\begin{array}{l}\text { Acute renal failure (ARF) } \\
105(69.1 \%)\end{array}$}} & Pre-renal & Volume depletion 29 (27.6\%) \\
\hline & & & $44(42 \%)$ & Sepsis or septic shock $15(14.3 \%)$ \\
\hline & & & \multirow{3}{*}{$\begin{array}{l}\text { Renal } \\
54(51.4 \%)\end{array}$} & Renal infiltration $6(5.7 \%)$ \\
\hline & & & & Tumor lysis syndrome 33 (31.4\%) \\
\hline & & & & Drug related $15(14.3 \%)$ \\
\hline & & & \multirow[t]{4}{*}{$\begin{array}{l}\text { Post-renal } \\
7(6.6 \%)\end{array}$} & Post renal obstruction $7(6.6 \%)$ \\
\hline & Hemorrhagic cystitis & $26(17$ & & \\
\hline & Nephrotic syndrome & $3(2 \%)$ & & \\
\hline & Renal masses & $18(11$ & & \\
\hline
\end{tabular}

TLS in the post-treatment period occurred in 12 patients (out of 33 patients diagnosed as ARF). Eleven patients had ALL and one patient had NHL. Serum uric acid levels ranged from $17-25 \mathrm{mg} / \mathrm{dL}$. 
Drugs inducing nephrotoxicity were: cisplatin (10 patients), amphotricin B (2 patients), acyclovir (1 patient), ifosfamide (1 patient), and aminoglycosides (1 patient).

Nephrotic syndrome following chemotherapy for HD was observed in 3 patients. Two of them were proved by renal biopsy to be of minimal change disease type and one showed secondary amyloidosis of the kidney.

Hemorrhagic cystitis complicated therapy with cyclophosphamide alone (10 patients), ifosfamide alone (6 patients) or both (10 patients).

Among the patients treated with a bone marrow transplant (total 18 patients), 7 patients (38.9\%) had renal complications: 5 patients in the early period after the bone marrow transplantation $\left(1^{\text {st }} 100\right.$ days) and 2 patients in the late period (after 100 days) and ( 3 patients had complications in both periods). In the early period (up to day 100), 2 patients (11.1\%) developed hypertension mostly related to drug toxicity (cyclosporine A) and/or microangiopathy and/or graft-versus-host disease-related complications. Three patients had persistent hypokalemia and two had persistent hypocalcemia that continued for long period in the post transplantation stage.

Table 3. Renal causes, management and outcome of acute renal failure (ARF) patients

\begin{tabular}{|c|c|c|c|}
\hline Causes & Number (\%) & & \\
\hline \multirow{6}{*}{$\begin{array}{l}\text { Tumor lysis syndrome } \\
\text { (TLS) } \\
\text { (33 patients) }\end{array}$} & & Pre-treatment & Post-treatment \\
\hline & Uric acid (mg/dL) & $11-55 \mathrm{mg} / \mathrm{dL}$ & $17-25 \mathrm{mg} / \mathrm{dL}$ \\
\hline & ALL & 14 & 11 \\
\hline & NHL & 6 & 1 \\
\hline & AML & 1 & 0 \\
\hline & Total cases & 21 & 12 \\
\hline \multirow{5}{*}{$\begin{array}{l}\text { Drug related } \\
\text { (15 patients ) }\end{array}$} & Cisplatin & 10 & \\
\hline & Amphotricin B & 2 & \\
\hline & Acyclovir & 1 & \\
\hline & Ifosfamide & 1 & \\
\hline & Aminoglycoside & 1 & \\
\hline \multirow{4}{*}{$\begin{array}{l}\text { Renal infiltration } \\
\text { ( } 6 \text { patients) }\end{array}$} & Bilateral Wilm's tumor & 1 & \\
\hline & ALL & 2 & \\
\hline & NHL & 2 & \\
\hline & Neuroblastoma & 1 & \\
\hline \multirow{3}{*}{$\begin{array}{l}\text { Management of all } \\
\text { patients with ARF (105) }\end{array}$} & Conservative & $79(75.2 \%)$ & \\
\hline & Acute dialysis & $18(17.1 \%)$ & \\
\hline & Chronic dialysis & $8(7.6 \%)$ & \\
\hline \multirow{3}{*}{$\begin{array}{l}\text { Outcome of all patients } \\
\text { with ARF (105) }\end{array}$} & Complete recovery & $70(66.7 \%)$ & \\
\hline & Chronic renal failure (CRF) & $8(7.6 \%)$ & \\
\hline & Death & $27(25.7 \%)$ & \\
\hline
\end{tabular}

Eighteen patients with ARF were treated with hemodialysis and/or hemofiltration (17.1\%). In patients of lesser degree of renal impairment, the presumed causative factors were eliminated, and the renal functions returned to normal.

As regard outcome of these cancer patients with renal involvement (152 patients), 70 of them (66.7\%) showed complete recovery of renal function, while 27 patients died (25.7\%). The remaining eight patients $(7.6 \%)$ were maintained on chronic replacement therapy. 
In the transplant patients, all patients were of minor impairment of renal function and most of them become normalized.

\section{Discussion}

In this study we evaluated all children with different types of malignancies in seven years period as regard renal involvement. Renal involvement occurred in about one fifth of all cancer patients 152/954 patients (15.9\%).

ARF is a serious complication of malignancies that causes substantial morbidity and mortality. Among critically ill cancer patients, $12 \%$ to $49 \%$ experience ARF and $9 \%$ to $32 \%$ require renal replacement therapy during their intensive care unit (ICU) stay ${ }^{[4]}$. In our study renal involvement was encountered in about $1 / 6(16 \%)$ of all children with cancer, of which the most common was ARF (69.1\% of all patients with renal involvement). Multiple causes leading to ARF in critically ill cancer patients are often present in combination as pre-renal failure [sepsis, dehydration or drugs (e.g., calcineurin inhibitors, ACE inhibitors. NSAIDs)], intrinsic renal failure [acute tubular necrosis secondary to ischaemia (shock or sepsis), or nephrotoxic agents (contrast agents, aminoglycosides, amphotricin, ifosfamide, cisplatin), disseminated intravascular coagulation, intravascular hemolysis, cancer infiltration (e.g., lymphoma, metastasis), glomerulonephritis or amyloidosis (acute leukemia, myeloma; renal carcinoma or HD)] and post-renal failure [Intra-renal obstruction (urate crystals, light chain, acyclovir, or methotrexate) or extrarenal obstruction (retroperitoneal fibrosis, urethral or bladder outlet obstruction] ${ }^{[4]}$. In our study different causes of ARF as (volume depletion, sepsis or septic shock, renal infiltration, TLC, drug toxicities or obstructive uropathy) were encountered (Table 2 and Table 3).

TLS is a potentially life-threatening complication of cancer treatment in patients with extensive, rapidly growing, chemosensitive malignancies. TLS results from the rapid destruction of malignant cells, which abruptly release intracellular ions, proteins and metabolites into the extra-cellular space. ARF may develop, the most common mechanism being uric acid crystal formation in the renal tubules secondary to hyper-uricemia. Another cause may be calcium phosphate deposition related to hyperphosphataemia ${ }^{[4]}$. TLS may occur spontaneously before treatment, but it usually develops shortly after the initiation of cytotoxic chemotherapy ${ }^{[5]}$. TLS mostly occurs in patients with high-grade hematological malignancies as Burkitt's lymphoma, lymphoblastic lymphoma, T-cell ALL and other acute leukemias ${ }^{[6]}$. ARF due to TLS occurred in 21 patients before treatment and 12 patients following treatment. It occurred in patients with ALL (25 patients), NHL (7 patients) and AML (1 patient).

Extra-renal obstruction causing ARF in cancer patients may nevertheless be present without hydronephrosis, during the first few days, when the collecting system is encased in retroperitoneal fibrosis or tumor or when the obstruction is partial ${ }^{[7]}$. In our study 7 patients had post-renal failure (obstructive uropathy) due to tumor compression by retroperitoneal rhabdomyosarcoma ( 2 patients), germ cell tumor (1 patient), paraspinal neuroblastoma ( 2 patients), and Burkitt's lymphoma (2 patients).

As regard renal toxicity related to cancer therapy, three drugs are reported to be commonly associated with ARF: cisplatin, ifosfamide and methotrexate ${ }^{[4]}$. Also cancer patients may be exposed to other potential nephrotoxic drugs such as antibiotics (aminoglycosides, vancomycin, amphotricin B), diuretics, anti-inflammatory drugs, analgetics, and radio-contrast media ${ }^{[2,8]}$. In our study Drugs inducing nephrotoxicity were: cisplatin (10 patients), amphotricin B (2 patients), acyclovir (1 patient), ifosfamide (1 patient), and aminoglycosides (1 patient).

Hemorrhagic cystitis is caused mainly by alkylating agents, such as cyclophosphamide and ifosfamide ${ }^{[9]}$. Hemorrhagic cystitis complicated therapy with cyclophosphamide and/or ifosfamide for encountered in 26 patients.

As regard BMT complications, ARF is among the most common potentially life-threatening complications. Occurrence rates of $30 \%$ to $84 \%$ have been reported. Sinusoidal obstruction syndrome (veno-occlusive disease of the liver) is the main cause of ARF in these patients ${ }^{[10,11]}$. However in this study, all the transplant patients with renal involvement were of 
minor impairment of renal function and most of them become normalized. In the early period (up to day 100), 2 patients (11.1\%) developed hypertension mostly related to drug toxicity (cyclosporine A) and/or microangiopathy and/or graft-versus-host disease-related complications. Three patients had persistent hypokalemia and two had persistent hypocalcemia that continued for long period in the post transplantation stage.

Prognosis for critically ill cancer patients is worse than the prognosis for critically ill patients without malignancy receiving renal replacement therapy ${ }^{[12]}$. In addition to hospital mortality, development of an ARF may affect optimal cancer treatment by requiring a decrease in chemotherapy dosage or by contraindicating potentially curative treatment ${ }^{[13]}$. As regard outcome of these cancer patients with renal involvement 27 patients died (25.7\%) and 8 patients $(7.6 \%)$ were maintained on chronic replacement therapy however most of the patients 70 of them $(66.7 \%)$ showed complete recovery of renal function.

ARF due to malignancy represented $14.7 \%$ of all patients of ARF in our pediatric nephrology unit, Mansoura University Children Hospital. Malignancy related chronic renal failure represented only $1.9 \%$ of all patients of chronic renal failure (306 patients) required chronic hemodialysis (unpublished data).

\section{Conclusion}

Renal involvement in children with cancer is a common problem. ARF is a common and severe complication. It results from various causes, including metabolic disturbances (TLS), renal infiltration by malignant cells, sepsis and drug induced toxicity. Prevention of ARF is mandatory in pediatric oncology units. Early prediction and anticipation of renal affection by frequent monitoring of renal functions especially in high risk patients. Prompt management by fluid expansion, alkalinization, and uricolytic treatment in patients with a high risk of acute TLS, elimination of nephrotoxic drugs, and monitoring of chemotherapy concentrations are among the measures which may reduce the risk of renal injury. A multidisciplinary approach is needed for adequate assessment, appropriate preventative measures and early intervention to reduce the incidence of life-threatening renal injury in cancer patients. All oncologists should keep in mind that they are not treating cancer only, but they are treating patient with cancer and should save the kidney as much as possible.

\section{References}

[1] Peterson WP, Reams R. Renal and electrolyte abnormalities due to chemotherapy. In: Perry MC (eds) The chemotherapy source book. Williams \& Wilkins, Baltimore. 1996; 727-744.

[2] Rossi R, Kleta R, Ehrich JHH. Renal involvement in children with malignancies. Pediatr Nephrol. 1999; $13: 153-162$. PMid:10229006 http://dx.doi.org/10.1007/s004670050585

[3] Lameire NH, Flombaum CD, Moreau D, Ronco C. Acute renal failure in cancer patients. Ann Med. 2005; $37: 13-25$. PMid:10229006 http://dx.doi.org/10.1080/07853890510007205

[4] Darmon M, Ciroldi M, Thiery G, Schlemmer B, Azoulay E. Clinical review: specific aspects of acute renal failure in cancer patients. Crit Care. 2006; 10: 211. http://dx.doi.org/10.1186/cc4907

[5] Jasek AM, Day HJ: Acute spontaneous tumor lysis syndrome. Am J Hematol. 1994; 47: 129-131. http://dx.doi.org/10.1002/ajh.2830470212

[6] Jeha S: Tumor lysis syndrome. Semin Hematol. 2001; Suppl 10: 4-8. http://dx.doi.org/10.1016/S0037-1963(01)90037-X

[7] Kapoor M, Chan GZ: Malignancy and renal disease. Crit Care Clin. 2001; 17: 571-598. http://dx.doi.org/10.1016/S0749-0704(05)70199-8

[8] Krawczuk-Rybak M, Kuźmicz M, Wysocka J. Renal function during and after treatment for acute lymphoblastic leukemia in children. Pediatr Nephrol. 2005; 20: 782-785. http://dx.doi.org/10.1007/s00467-005-1839-3

[9] Schuchter LM, Hensley ML, Meropol NJ, Winer EP, American Society of Clinical Oncology Chemotherapy and Radiotherapy Expert Panel: 2002 update of recommendations for the use of chemotherapy and radiotherapy protectants: clinical practice guidelines of the American Society of Clinical Oncology. J Clin Oncol. 2002; 20: 2895-2903.

http://dx.doi.org/10.1200/JCO.2002.04.178 
[10] Noel C, Hazzan M, Noel-Walter MP, Jouet JP: Renal failure and bone marrow transplantation. Nephrol Dial Transplant. 1998; 13: 2464-2466. http://dx.doi.org/10.1093/ndt/13.10.2464

[11] Zager RA, O’Quigley J, Zager BK, Alpers CE, Shulman HM, Gamelin LM, Stewart P, Thomas ED: ARF following bone marrow transplantation: a retrospective study of 272 patients. Am J Kidney Dis. 1989; 13: 210-216. PMid:2645771

[12] Benoit DD, Depuydt PO, Vandewoude KH, Offner FC, Boterberg T, De Cock CA, Noens LA, Janssens AM, Decruyenaere JM: Outcome in critically ill medical patients treated with renal replacement therapy for acute renal failure: comparison between patients with and those without haematological malignancies. Nephrol Dial Transplant. 2005; 20: 552-558. PMid:15671075 http://dx.doi.org/10.1093/ndt/gfh637

[13] Munker R, Hill U, Jehn U, Kolb HJ, Schalhorn A. Renal complications in acute leukemias. Haematologica. 1998 ; 83: $416-421$. PMid:9658725 